\title{
Adolescent Psychological Health and Quality Education under the New Situation
}

\author{
Kai Yu \\ Jiangxi Manufacturing Polytechnic College,Nanchang,Jiangxi province, China,330095
}

\begin{abstract}
:
This paper, aiming at the psychological health and quality of contemporary adolescents, discusses the specific integration method from three aspects based on the structural composition and evaluation mode of adolescent's psychological quality, and then carries on a simple research on the integration of psychological health and quality education.
\end{abstract}

Keywords: adolescent; psychological health; psychological quality

\section{Introduction}

In recent times, economic globalization has been developing deeply and broadly and science and technology has been progressing with each passing day, as a consequence, cultures and values in different countries may interact and collide mutually and international competition becomes more and more obvious.

Competition in comprehensive national power is the competition of talent and national quality competition in the final analysis. Adolescents' psychological quality, not only affects their own development, but also relates to the improvement of national quality, which plays a significant role in the cultivation of the talents in the new century and the great rejuvenation of the Chinese nation. According to relevant data, There are nearly 1/5 children in China are suffering from psychological distress among 340 million Chinese teenagers. The Chinese Centers for Disease Control and Prevention and Mental Health Center points out that the number of Chinese children under the age of 17 affected by various mental disorders and behavior problems reach up to 50 million.

The illegal and criminal activities of adolescents are frequently reported by newspapers, such as murder and injury case, which shows that the mental health condition of adolescents at present is not optimistic, so, it is of great necessity and urgency to strengthen the mental health education and psychological quality training of contemporary adolescents. It is in this case that this paper carries out the integrated study on the adolescent mental health and psychological quality training of adolescents, in order to make the scientific and effective contribution to our country's burgeoning adolescent mental health education and psychological quality cultivation and through the exploratory research.

\section{Analysis of theoretical basis of the integrated researches}

\subsection{The relationship between psychological health and quality}

Psychological health and psychological quality are two important concepts which appear frequently in psychological health education in China, therefore, the basic definitions and their relationship with each other must be clarified.

In terms of the exceedingly pessimistic and negative psychological state, there still exists deficiencies such as diagnostic uncertainty, therefore, the related researchers in western countries put forward a kind of double-factor model of psychological health taking psychological health state as the core.

In this model, the subjective well-being dominating positive psychological health index and the psychological illness dominating negative mental health indicator are listed into the evaluation indexes of psychological health, therefore, it makes up the unilateral view in terms of psychological illness. But fully evaluation of the psychological health states of the 
individual is only applicable to the common psychological health diagnosis, which is difficult to fundamentally promote psychological health of the human being.

The author makes a summary of the researches on psychological health and psychological quality in recent years and, on this basis, the relationship between psychological health and psychological quality is directly found out. If the relationship between the two concepts is set up as a model, including the internal mechanism and external behavior, for individual psychological health, the latter one is decided by the former one.

\subsection{The interaction between adolescent psychological health and quality}

Adolescent psychological health and quality education is a very complex system, which has the close relation between each other and influences each other. Based on a mass of theories and researches, this paper puts forward the interaction theory of the adolescent psychological health and psychological quality. The psychology of adolescent changes quickly and unpredictably, and the change of the social reality is also in the ongoing. With the adolescent psychology and social reality blending together, it leads to a series of psychological problems of the contemporary adolescents.

Therefore, in the psychological health education of contemporary adolescent, at the same time, a thorough investigation should be done on the relationship between the subjective and objective factors, and strategies should be adopted to strengthen the external and internal psychological quality through the interaction between subjective and objective viewpoints.

In addition, from the adaption to the realistic social environment and the urgent requirement for adolescent psychological health development, the frequency and characteristic of adolescent psychological health problems should be accurately grasped, and interactive study on the adaption and development of adolescents, and balance and conflict should also be conducted, so as to explore educational strategies for adolescent psychological health problem from multiple levels. At last, based on the relationship between psychological health and psychological quality, this paper puts forward how to effectively integrate adolescent psychological health and psychological quality and handle with the nature of the relationship between the two appropriately.

\section{The structure composition and evaluation mode of adolescent psychological quality}

On the measurement and evaluation of adolescent psychological health, there has been a mature method in foreign countries, but the psychological quality is a concept newly proposed in recent years, whose measurement and evaluation method has not yet formed a standard system and still needs further discussion.

In terms of the basic definition of psychological quality, this paper works out the following definition based on the related researches at home and abroad and combining the relevant practical experience in recent years: psychological quality is a hidden, stable and inner psychological quality based on the physiological condition and external environment and has functions of derivation and development. To be brief, psychological quality is a stable quality in the inner nature of human, whose formation is stable process from eternal stimulation to inner quality which is embodied through the daily behavior habits of human beings.

Psychological quality is composed of cognitive quality, personality and adaptability. The cognitive quality refers to the psychological quality of individual embodied in the process of cognition; Personality refers to a combination of a variety of psychological characteristics, including characteristics of motivation, feature and mood; Adaptability refers to the adaption to the external environment and internal environment.

\section{Principal psychological problems of adolescents}

The main theme of the integrated study on psychological health and psychological quality is to explore the principal psychological problems of contemporary adolescent groups, which is the window to carry out a reasonable school psychological health 
education. After the author's investigation and analysis, the following main problems are summarized.

\subsection{Psychological pressure}

The most common problems in adolescent group is psychological pressure, and the source of the psychological pressure usually comes from academics and social aspects. The author uses the comprehensive orientation and analyzed the collected the main measures adolescents take in face of pressure. In addition, the author also analyzes the relationship between the pressure source and measures the form directly, so as to build a set of perfect models to cope with pressure.

\subsection{Adaption to learning}

In addition to psychological pressure, adaptation to learning is also one of the major problems adolescents face with. In the process of learning, adolescent students will inevitably form an intangible comparison with other students. The psychology will result in increasing pressure on students' in learning, that is, they cannot adapt to the group. The author, through the investigation, concludes some common types of adolescents' adaptation to learning, for example, exam psychological problems.

\subsection{Interpersonal relationship}

Interpersonal relationship is common problem that exists in the adolescent group, which is mainly from the competition among group members, heterosexual contacts, parent-child communication, etc.. In the process of these relationships, the slightly improper behaviors will easily lead to psychological problems. Based on the study on groups that adolescent frequently contact with in daily communication, such as students, members of opposite sex, parents and so on, problems prone to occur in these different groups are analyzed in the process of communication, and the corresponding solution countermeasures are also made out as a matter of course.

\subsection{Emotional problems}

The author, through studying on some common emotional problems of adolescents, summarizes the development process and characteristics of adolescent emotional problems. The study begins with the physical arousal of fear, fear of psychological activity and others evaluation, and discusses the effects of the development characteristics of adolescent anxiety and sensitive mood on the adolescent. In addition, the author also explores characteristics and causes of depressive symptoms and depression factors, and then analyzes the specific countermeasures.

\subsection{Psychology of love}

The psychological change presenting in love is also one of the main features of the adolescent psychological development, therefore, the author establishes the concepts associated with adolescent's love, and explores the attitude of adolescents towards love and the development characteristics of their psychology through the investigation. From the viewpoint of love psychology and pressure source, compiles a set of test forms to explore the love psychological problems and proposes the contemporary processing method and regulation strategy for Chinese adolescents to eliminate the love pressure.

\subsection{Occupational health}

The author, aiming at the present psychological state of Chinese adolescents in occupational health, explores adolescents' occupational psychological quality, and establishes a set of local structure mode from the perspective of career development, so as to develop the corresponding measurement and evaluation tools. Thus, the author analyzes the development trend and characteristics of the Chinese adolescents in the occupation development, and studies the important factors that affect psychological problems in occupational development.

\section{Conclusion}

Specific integrated strategies for adolescent psychological health and quality education:The integrated education of adolescent psychological health and psychological quality aims to adapt to the adolescent psychological health and quality course in Chinese schools. So far, there are no ready-made study methods and standards. Therefore, we should always follow the following principles in the research process. 


\subsection{Stick to "person-in-environment" theory}

The idea" person-in-environment is proposed during the process of reflecting the research on the traditional psychological health education, whose main theoretical basis has the following several aspects: Any kind of human psychological activities has close relation with the society from the perspective of people's senior psychological and social relations, such as thinking, personality, emotion and so on, integrated research on adolescent psychological health and psychological quality must be combined with social environment factors. A comprehensive analysis on adolescent psychological health and psychological quality from the perspective of environment can promote the harmonious development of the individual and the environment effectively.

On the other hand, human is a combination of many physical and psychological factors, and physical and nonphysical factors influence each other. In addition to congenital genetic defects, any psychological problems are affected by the external environment, such as interpersonal relationship, working pressure, study pressure, economic condition, and so on. Therefore, in the integration of research on adolescent psychological health and psychological quality education, psychological problems and non-psychological problems must be commonly discussed. Only in this way can we truly achieve the goal of mental health.

\subsection{Keep to scientific and reasonable principle}

The scientific and reasonable method mainly refers to the scientific fundamental theory and method, but this principle is selectively applied. Adolescent psychological health is affected by many factors, therefore, in the process of research, we must emphasize on the scientificity and rationality. The correct use of the efficient principle in modern psychological science can assure the scientificity and rationality of the study. On the other hand, the study should be combined with psychological development features of contemporary adolescents from the aspects of social environment, and multi-level multi-angle exploration can be conducted aiming at the integration of adolescent psychological health and the cultivation of the adolescents' psychological quality.

\section{Reference:}

[1]Zhu Jun. Looking for spiritual home for children [J]. Procuratorial View, 2006,8(11)

[2]Zhang Xiangkui, Li Lihong.. psychology of adolescence $[\mathrm{M}]$.Chang Chun: Journal of Northeast Normal University Press, 2005:22

[3]Zhang Dajun. Overview of psychological quality education in school [M].Chong Qing Southwest China Normal University Press, 2004 